Chryseomonas luteola. L'antibiogramma parallelamente eseguito dimostrò sensibilità del ceppo nei confronti di diverse classi di antibiotici (aminoglucosidi, beta-lattamine, carbapenemici ecc.). In seguito a terapia con ceftazidime, il paziente migliorò gradualmente sino ad essere dimesso apparentemente guarito dopo 15 giorni .

Descriviamo il caso per l'assoluta rarità delle segnalazioni di Chryseomonas luteola in patologia umana .Questo da noi segnalato è uno dei pochi casi descritti di infezione cutanea da Chryseomonas luteola.

\title{
032
}

\section{DESCRIZIONE DI UN CASO DI ASCESSO CUTANEO CAUSATO DA CHRYSEOMONAS LUTEOLA}

Fazii P.'; Pelatti A.'; Civitarese C. ${ }^{2}$; Russi C.'; Pistola F.'; Stella M.'; Crescenzi C. '; Gattone M.C.'; Manes E.'; Colagrande E.'; Riario Sforza G.' .

' U.O.di Laboratorio Analisi Chimico-cliniche e Micro-biologia , 2 U.O. di Ortopedia P.O. "Spirito Santo",

Via Fonte Romana 8, - 65124 Pescara

Chryseomonas luteola appartenente alla famiglia delle Pseudomonaceae è un bacillo gram negativo, aerobio obbligato, non fermentante, ossidante che si sviluppa bene sui comuni terreni di coltura dove forma un pigmento di colore giallo-oro. Chryseomonas luteola non si rinviene facilmente a livello ambientale, ma è commensale dell'uomo e può essere isolata in vari distretti, cute inclusa. Chryseomonas luteola è stato associato a batteriemie, endocarditi, ascessi, peritoniti, senza però che fosse stato stabilito, in questi casi, un sicuro ruolo patogeno; probabilmente si comporta da patogeno opportunista. Descriviamo il caso di un ascesso cutaneo sostenuto da Chryseomonas luteola in un soggetto di 63 anni, residente nel pescarese, contadino. Egli, nel mese di maggio 2004, mentre attendeva ai lavori di campagna, si arrecava con un coltello a lama lunga, una profonda ferita dei tessuti molli a livello del II dito della mano sinistra. L'edema imponente, la febbre ed il forte dolore consigliarono i familiari a ricoverarlo presso l'U.O. di Ortopedia del nostro nosocomio. Gli accertamenti radiologici non mostrarono un coinvolgimento osseo; possibile era però il rischio che la profonda lesione ascessualizzata avrebbe potuto comportare una complicanza osteomielitica. Prima di trattare il paziente con una terapia empirica, fu inviato in Laboratorio un tampone della ferita. Sui comuni terreni di coltura, si svilupparono grandi colonie gialle che, mediante il sistema Vitek - bioMerieux, furono identificate come appartenenti alla specie 